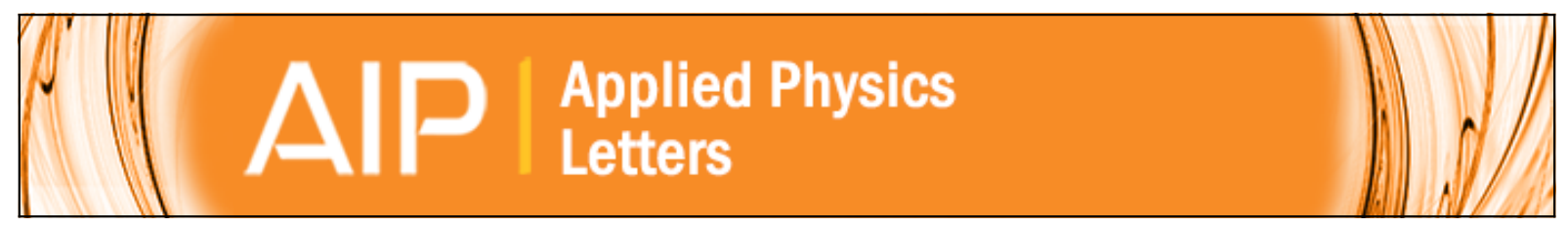

Terahertz transmission characteristics of high-mobility GaAs and InAs twodimensional-electron-gas systems

N. A. Kabir, Y. Yoon, J. R. Knab, J.-Y. Chen, A. G. Markelz, J. L. Reno, Y. Sadofyev, S. Johnson, Y.-H. Zhang, and J. P. Bird

Citation: Applied Physics Letters 89, 132109 (2006); doi: 10.1063/1.2357605

View online: http://dx.doi.org/10.1063/1.2357605

View Table of Contents: http://scitation.aip.org/content/aip/journal/apl/89/13?ver=pdfcov

Published by the AIP Publishing

Articles you may be interested in

The influence of charged InAs quantum dots on the conductance of a two-dimensional electron gas: Mobility vs. carrier concentration

Appl. Phys. Lett. 99, 223510 (2011); 10.1063/1.3665070

High-mobility window for two-dimensional electron gases at ultrathin AI N/Ga N heterojunctions

Appl. Phys. Lett. 90, 182112 (2007); 10.1063/1.2736207

High-mobility two-dimensional electron gas in In Al As/In As heterostructures grown on virtual InAs substrates by molecular-beam epitaxy

Appl. Phys. Lett. 90, 012115 (2007); 10.1063/1.2430403

Two-dimensional electron-gas actuation and transduction for $\mathrm{GaAs}$ nanoelectromechanical systems

Appl. Phys. Lett. 81, 3879 (2002); 10.1063/1.1516237

Transport properties of two-dimensional electron gases containing InAs self-assembled dots

Appl. Phys. Lett. 73, 2468 (1998); 10.1063/1.122484 


\title{
Terahertz transmission characteristics of high-mobility GaAs and InAs two-dimensional-electron-gas systems
}

\author{
N. A. Kabir and Y. Yoon \\ Department of Electrical Engineering, University at Buffalo, The State University of New York, Buffalo, \\ New York 14260-1920 \\ J. R. Knab, J.-Y. Chen, and A. G. Markelz ${ }^{\text {a) }}$ \\ Department of Physics, University at Buffalo, The State University of New York, Buffalo, \\ New York 14260-1920 \\ J. L. Reno \\ Nanostructure \& Semiconductor Physics Department, Sandia National Laboratories, P.O. Box 5800, \\ Albuquerque, New Mexico 87185-1415 \\ Y. Sadofyev, S. Johnson, and Y.-H. Zhang \\ Department of Electrical Engineering, Arizona State University, Tempe, Arizona 85287-5706 \\ and Center for Solid State Electronics Research, Arizona State University, Tempe, Arizona 85287-5706 \\ J. P. Bird ${ }^{\text {b) }}$ \\ Department of Electrical Engineering, University at Buffalo, The State University of New York, Buffalo, \\ New York 14260-1920
}

(Received 16 May 2006; accepted 2 August 2006; published online 26 September 2006)

\begin{abstract}
Frequency-dependent complex conductivity of high-mobility GaAs and InAs twodimensional-electron-gas (2DEG) systems is studied by terahertz time domain spectroscopy. Determining the momentum relaxation time from a Drude model, the authors find a lower value than that from dc measurements, particularly at high frequencies/low temperatures. These deviations are consistent with the ratio $\tau_{t} / \tau_{q}$, where $\tau_{q}$ is the full scattering time. This suggests that small-angle scattering leads to weaker heating of 2DEGs at low temperatures than expected from dc mobility.

(C) 2006 American Institute of Physics. [DOI: 10.1063/1.2357605]
\end{abstract}

There is significant interest in developing terahertz sources and detectors that utilize high-mobility twodimensional-electron-gas (2DEG) systems. ${ }^{1-6}$ In this letter, we therefore use terahertz time domain spectroscopy (TDS) to measure the ac conductivity of GaAs and InAs 2DEGs. Terahertz TDS allows for contactless measurement of the frequency-dependent $\left(\sim 5-85 \mathrm{~cm}^{-1}\right)$ complex conductivity by coherently detecting the transmitted amplitude and phase shift of a laser-generated electric-field pulse. In such studies, it is often assumed that free-carrier absorption is described by a Drude model, using the relaxation time obtained from dc transport. The optical conductivity of metal films has been found to be best described by a "modified Drude model," however, that incorporates a frequency-dependent scattering time. ${ }^{7}$ A similar model has also been used in studies of terahertz conductivity in transient photoconductivity of bulk GaAs. ${ }^{8}$

In this letter, we show that a modified Drude model fits the measured terahertz complex conductivity of GaAs and InAs 2DEGs, with relaxation times $\left(\tau_{\mathrm{THZ}}\right)$ shorter than those from dc mobility $\mu$, which yields the mobility lifetime $\tau_{t}$. This discrepancy is increasingly pronounced with decreasing temperature $(T)$. Near $4.2 \mathrm{~K}$, our analysis suggests that $\tau_{\mathrm{THz}}$ is actually intermediate between $\tau_{t}$ and the quantum lifetime $\tau_{q}$, which defines the total-scattering rate, extracted from analysis of the Shubnikov-de Haas ( $\mathrm{SdH}$ ) effect. We suggest that, due to the role of small-angle scattering, Drude heating

\footnotetext{
a) Author to whom correspondence should be addressed.

${ }^{b)}$ Electronic mail: jbird@buffalo.edu
}

of high-mobility 2DEGs by terahertz radiation can be significantly weakened at low $T$, compared to estimates based on dc transport. These results are therefore relevant for terahertz photonic-device development since they reveal that one cannot use dc transport to infer free-carrier loss.

In terahertz TDS, the sample transmission is referenced to that of a substrate (we use a piece of the same heterostructure with its 2DEG layer removed by etching). Its complex conductivity is then related to the transmission characteristics by the thin-film approximation, ${ }^{9}$

$$
t=\frac{E_{\text {film }} e^{i \varphi_{\text {film }}}}{E_{\text {sub }} e^{i \varphi_{\text {sub }}}}=\frac{t_{\text {film }}}{t_{\text {sub }}}=\frac{1+n_{\text {sub }}}{1+n_{\text {sub }}+Z_{0} \sigma_{s}},
$$

where

$$
\begin{gathered}
\sigma_{s}=\sigma_{s}^{(1)}+i \sigma_{s}^{(2)}, \quad \operatorname{Re}\left[\frac{1}{t}\right]=1+\frac{Z_{0} \sigma_{s}^{(1)}}{1+n_{\text {sub }}}, \\
\operatorname{Im}\left[\frac{1}{t}\right]=\frac{Z_{0} \sigma_{s}^{(2)}}{1+n_{\text {sub }}}
\end{gathered}
$$

Here, $E_{\text {film }}\left(E_{\text {sub }}\right)$ is the magnitude of the electric field transmitted by the film (substrate), $t_{\text {film }}\left(t_{\text {sub }}\right)$ is the complex terahertz transmission of the film (substrate), $Z_{0}$ is the characteristic impedance of free space $(\sim 377 \Omega)$, and $n_{\text {sub }}[=3.3$ for GaAs (Ref. 10)] is the refractive index of the substrate. In the modified Drude model, the complex ac conductivity at frequency $\omega,{ }^{11}$ 
TABLE I. Summary of the key parameters of the GaAs and InAs 2DEG samples.

\begin{tabular}{lcccccccc}
\hline \hline & $\begin{array}{c}\text { Hall } n_{s} \text { at } \\
300 \mathrm{~K} \\
\left(10^{15} \mathrm{~m}^{-2}\right)\end{array}$ & $\begin{array}{c}\text { Hall } n_{s} \text { at } \\
4.2 \mathrm{~K} \\
\left(10^{15} \mathrm{~m}^{-2}\right)\end{array}$ & $\begin{array}{c}\mathrm{SdH} n_{s} \text { at } \\
\begin{array}{c}4.2 \mathrm{~K} \\
\left(10^{15} \mathrm{~m}^{-2}\right)\end{array}\end{array}$ & $\begin{array}{c}\mu \text { at } \\
\left(\mathrm{cm}^{2} / \mathrm{V} \mathrm{s}\right)\end{array}$ & $\begin{array}{c}\mu \text { at } \\
\left(\mathrm{cm}^{2} / \mathrm{K} \mathrm{s}\right)\end{array}$ & $\begin{array}{c}\tau_{t} \text { at } \\
(\mathrm{ps})\end{array}$ & $\begin{array}{c}\tau_{q} \text { at } \\
(2 \mathrm{~K} \\
(\mathrm{ps})\end{array}$ & $\begin{array}{c}\tau_{t} / \tau_{q} \text { at } \\
4.2 \mathrm{~K}\end{array}$ \\
\hline GaAs & 8.1 & 2.3 & 2.3 & 7000 & 2000000 & 76 & 0.14 & 540 \\
InAs & 84 & 79 & $36^{\mathrm{a}}, 26^{\mathrm{b}}$ & 21000 & 450000 & 1.4 & 0.10 & 14 \\
\hline
\end{tabular}

${ }^{a}$ Density due to first 2DEG subband.

${ }^{\mathrm{b}}$ Density due to second 2DEG subband.

$$
\sigma_{s}=\frac{n_{s} e^{2} \tau}{m^{*}(\omega)} \frac{1}{1-i \omega \tau(\omega)},
$$

where $n_{s}$ is the 2DEG carrier density, $m^{*}(\omega)$ is the frequencydependent effective mass, and $\tau(\omega)$ is the frequencydependent relaxation time. A strength of terahertz TDS is that one can directly extract $\tau(\omega)$ (which we will refer to hereafter as $\left.\tau_{\mathrm{THZ}}\right)$, independent of $m^{*}(\omega)$ and $n_{s}$,

$$
\tau_{\mathrm{THz}}=\frac{\sigma_{s}^{(2)}}{\omega \sigma_{s}^{(1)}}=\frac{1}{\omega[\operatorname{Re}(1 / t)-1]} .
$$

GaAs and InAs 2DEGs were investigated in this study. The GaAs 2DEG is comprised of a $10 \mathrm{~nm}$ undoped GaAs cap layer, $98 \mathrm{~nm}$ of undoped AlGaAs, $2 \mathrm{~nm}$ of undoped AlAs, $2.3 \mathrm{~nm}$ of undoped GaAs, a Si delta-doped layer with an areal density of $10^{12} \mathrm{~cm}^{-2}, 0.6 \mathrm{~nm}$ of undoped GaAs, $2 \mathrm{~nm}$ of undoped AlAs, a $75 \mathrm{~nm}$ AlGaAs spacer layer, a $30 \mathrm{~nm}$ GaAs quantum well, a $95 \mathrm{~nm} \mathrm{AlGaAs} \mathrm{lower} \mathrm{spacer}$ layer, $2 \mathrm{~nm}$ of undoped AlAs, $2.3 \mathrm{~nm}$ of undoped GaAs, a lower $\mathrm{Si}$ delta-doped layer with an areal density of $10^{12} \mathrm{~cm}^{-2}, 0.6 \mathrm{~nm}$ of undoped GaAs, $2 \mathrm{~nm}$ of undoped AlAs, $98 \mathrm{~nm}$ of undoped AlGaAs, 300 periods of a GaAs/AlGaAs smoothing superlattice, and a 100-nm-thick undoped GaAs buffer. The InAs 2DEG (Ref. 12) was grown on GaAs and was comprised of a $6 \mathrm{~nm} \mathrm{GaSb}$ cap layer, $25 \mathrm{~nm}$ of undoped $\mathrm{AlSb}$, a Te delta-doped layer, a $15 \mathrm{~nm}$ undoped AlSb spacer layer, a $15 \mathrm{~nm}$ InAs quantum well, a $15 \mathrm{~nm}$ AlSb spacer layer, a Te delta-doped layer, a $20 \mathrm{~nm}$ undoped AlSb layer, ten periods of a $2.5 \mathrm{~nm} \mathrm{AlSb} / 2.5 \mathrm{~nm} \mathrm{GaSb}$ smoothing superlattice, and undoped buffer layers of $2.5 \mathrm{~mm}$ AlSb, $100 \mathrm{~nm} \mathrm{AlAs}$, and $200 \mathrm{~nm}$ GaAs. For transport measurements, GaAs Hall bars with alloyed contacts were formed by optical lithography and lift-off. The InAs 2DEG was cleaved into small pieces and alloyed contacts were formed by In soldering.

$\mathrm{SdH}$ and Hall effects were measured in the GaAs and InAs 2DEGs at 1.4-40 K. These measurements were made by standard lock-in techniques and yield information on the $T$ dependence of $\tau_{t}$. An analysis of the $\mathrm{SdH}$ oscillations also allows $\tau_{q}$ to be determined. ${ }^{13}$ Table I lists the parameters extracted for the 2DEGs. The different $n_{s}$ extracted for InAs from the $\mathrm{SdH}$ and Hall effects indicate partial filling of a second subband. Due to nonparabolicity of the InAs conduction band, the dc effective mass was extracted from the temperature dependence of the $\mathrm{SdH}$ oscillations. ${ }^{13}$ This gave $m^{*}=0.060 m_{0} \pm 0.005 m_{0}$, in good agreement with the predictions of the Kane model for this density. ${ }^{14}$ The main uncertainty in our analysis is in our estimate of $\tau_{q}$. The error bars in Fig. 3 show the range of $\tau_{q}$ obtained from our analysis, but even after considering this uncertainty there is still some (a factor of 2) discrepancy between the turn-on field of the $\mathrm{SdH}$ oscillations and the low-temperature value of $\tau_{q}$. This does not change the quantitative conclusions of our work, however.

Terahertz TDS (Fig. 1) was performed in a cryostat system cooled to $10 \mathrm{~K}$. The system uses transient current generation from an antenna on semi-insulating $\mathrm{GaAs}$ and electro-optic detection. A $65 \mathrm{fs}, 350 \mathrm{~mW}$, and $82 \mathrm{MHz}$ Ti:sapphire laser was used to generate/detect the terahertz pulse. The cryostat has two sets of terahertz-transparent polyethylene windows in the path of the terahertz beam. The scan resolution was $0.02 \mathrm{THz}$ and the time-domain data were Fourier transformed to give the magnitude/phase of the transmitted field for the sample and reference substrate. The bandwidth is $2.5 \mathrm{THz}$, but since the absorbance falls off dramatically above $0.5 \mathrm{THz}$ we emphasize here the range over which the frequency dependence is apparent. Transmission $(|t|)$ and phase $\left[\tan ^{-1}(\operatorname{Im}(t) / \operatorname{Re}(t))\right]$ of the $\operatorname{InAs}$ and GaAs 2DEGs are shown in Fig. 1. These data are plotted as discrete points, and are averaged values calculated over a $0.1 \mathrm{THz}$ window. The high $n_{s}$ of the InAs 2DEG is responsible for its stronger absorption (particularly below $1 \mathrm{THz}$ ) compared to the GaAs system.

Figure 2 shows the frequency dependence of $\tau_{\mathrm{THz}}$ [from Eq. (3)] for the 2DEGs at several temperatures. At $300 \mathrm{~K}$, $\tau_{\mathrm{THz}}$ appears to be independent of frequency. At lower tem-

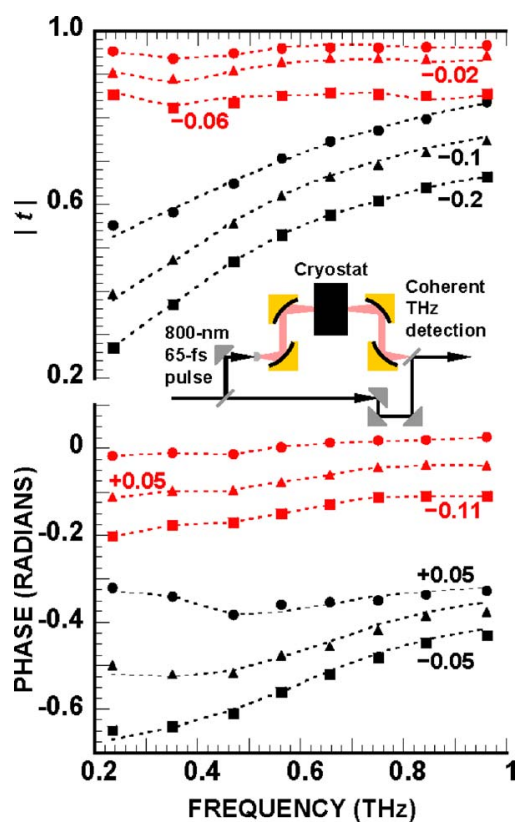

FIG. 1. (Color online) Field transmission magnitude (upper panel) and phase (lower panel) of the GaAs (red data) and InAs (black data) 2DEGs. The solid symbols are the original experimental data. The dotted lines are fits to Eq. (2) using the variation of $\tau_{\mathrm{THz}}$ inferred from the thin-film approximation [E. (3)] Circles $300 \mathrm{~K}$ triangles: $125 \mathrm{~K}$, and squares: $20 \mathrm{~K}$. Inset: schematic of the key features of the terahertz TDS system. 

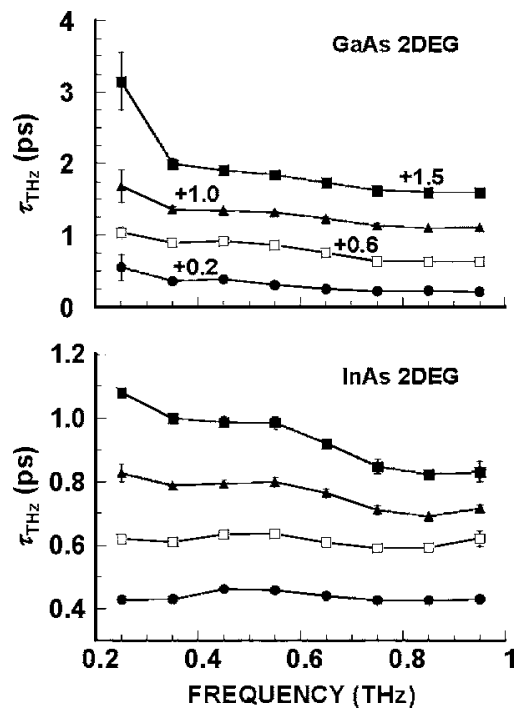

FIG. 2. Frequency dependence of $\tau_{\mathrm{THz}}$ inferred from the thin-film approximation [Eq. (3)]. Circles: $300 \mathrm{~K}$, open squares: $200 \mathrm{~K}$, triangles: $125 \mathrm{~K}$, and filled squares: $20 \mathrm{~K}$. The solid lines are interpolated between data points.

peratures, however, $\tau_{\mathrm{THz}}$ shows a significant decrease with increasing frequency. Similar behavior has been reported in midinfrared measurements of metal films. ${ }^{7}$ In Fig. 1, we have substituted the frequency-dependent values of $\tau_{\mathrm{THz}}$ into Eq. (2) to calculate the transmission amplitude/phase and we plot these results as the dotted lines. We have used $m_{\text {InAs }}^{*}$ $=0.06 m_{0}$ and $m_{\mathrm{GaAs}}^{*}=0.067 m_{0}$ in these calculations, along with the total 2DEG densities (Table I). The fits show good agreement with experiment, with small deviations arising from the frequency dependence in the effective mass.

Figure 3 shows the various lifetimes obtained from our measurements. Near $300 \mathrm{~K}$, the dc mobility is limited predominantly by electron-phonon scattering. ${ }^{15}$ As the temperature is lowered below this, the values of $\tau_{t}$ and $\tau_{\mathrm{THz}}$ differ significantly. This is clearest for the GaAs 2DEG in which, at the lowest temperatures, $\tau_{\mathrm{THz}}$ is several orders of magnitude smaller than $\tau_{t}$ but close to $\tau_{q}$. The difference between $\tau_{q}$ and $\tau_{t}$ in such 2DEGs is known ${ }^{16}$ to result from the predominance of small-angle scattering at low-temperatures, which does not significantly limit the mobility. The large ratio of $\tau_{t} / \tau_{q}$ in the GaAs 2DEG is due to its thick spacer layers. The suggestion therefore is that at low temperatures, small-angle scattering results in reduced terahertz absorption, relative to the value expected from the dc mobility.

Previously, the modified Drude model was shown to agree well with terahertz measurements of bulk GaAs. ${ }^{8}$ Our analysis suggests a different picture to describe the interaction of terahertz radiation with a high-mobility 2DEG. Near $300 \mathrm{~K}$, where electron-phonon scattering should dominate the mobility, ${ }^{15}$ the results of our experiment approach the predictions of the dc Drude model. At low $T$, however, where impurity scattering dominates, large-angle scattering predominantly limits the dc mobility while the terahertz transmission seems to be sensitive to the total-scattering $\left(\tau_{q}\right)$ rate, particularly above $\sim 0.5 \mathrm{THz}$. Since $\tau_{t} / \tau_{q}$ can be very large ( $>100$ for GaAs), low-temperature Drude heating of 2DEGs by terahertz radiation can be significantly weaker than simple estimates based on the 2DEG mobility. Our measurements of the InAs 2DEG are consistent with this idea. The ratio $\tau_{t} / \tau_{q}$ for this $2 \mathrm{DEG}$ is nearly 40 times smaller than that

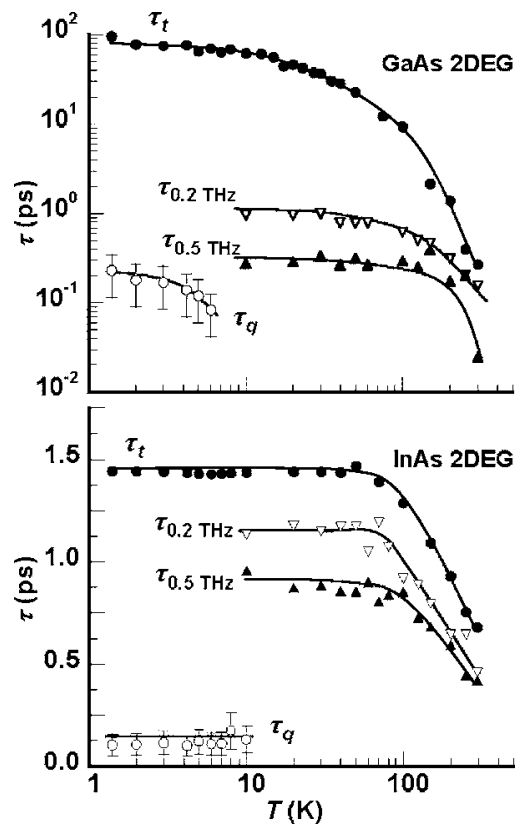

FIG. 3. Temperature dependence of $\tau_{\mathrm{THz}}, \tau_{t}$, and $\tau_{q}$ in the GaAs (upper) and InAs (lower) 2DEGs. The solid lines are guides for the eyes.

in GaAs (see Table I), and the discrepancy between $\tau_{\mathrm{THz}}$ and $\tau_{t}$ is also less pronounced (Fig. 3). The smaller value of $\tau_{t} / \tau_{q}$ reflects the fact that the InAs 2DEG has no spacer layers and is unintentionally doped by donors that are believed to be located close to the interfaces of the quantum well.

The work at Buffalo is supported by the Department of Energy and NYSTAR. Sandia is a multiprogram laboratory operated by Sandia Corporation, a Lockheed Martin Company, for the United States Department of Energy's National Nuclear Security Administration under Contract No. DEAC04-94AL85000.

${ }^{1}$ X. G. Peralta, S. J. Allen, M. C. Wanke, N. E. Harff, J. A. Simmons, M. P. Lilly, J. L. Reno, P. J. Burke, and J. P. Eisenstein, Appl. Phys. Lett. 81, 1627 (2002).

${ }^{2}$ E. A. Shaner, M. Lee, M. C. Wanke, A. D. Grine, J. L. Reno, and S. J. Allen, Appl. Phys. Lett. 87, 193507 (2005).

${ }^{3}$ W. Knap, Y. Deng, S. Rumyantsev, J.-Q. Lu, M. S. Shur, C. A. Saylor, and L. C. Brunel, Appl. Phys. Lett. 80, 3433 (2002).

${ }^{4}$ W. Knap, Y. Deng, S. Rumyantsev, and M. S. Shur, Appl. Phys. Lett. 81, 4637 (2002).

${ }^{5}$ I. V. Kukushkin, S. A. Mikhailov, J. H. Smet, and K. von Klitzing, Appl. Phys. Lett. 86, 044101 (2005).

${ }^{6}$ P. S. Dorozhkin, S. V. Tovstonog, S. A. Mikhailov, I. V. Kukushkin, J. H. Smet, and K. von Klitzing, Appl. Phys. Lett. 87, 092107 (2005).

${ }^{7}$ J. Cerne, D. C. Schmadel, M. Grayson, G. S. Jenkins, J. R. Simpson, and H. D. Drew, Phys. Rev. B 61, 8133 (2000).

${ }^{8}$ M. C. Beard, G. M. Turner, and C. A. Schmuttenmaer, Phys. Rev. B 62, 15764 (2000).

${ }^{9}$ S. W. McKnight, K. P. Stewart, H. D. Drew, and K. Moorjani, Infrared Phys. 27, 327 (1987).

${ }^{10}$ O. Madelung, Semiconductors: Basic Data, 2nd ed. (Springer, Berlin, 1996), pp. 109-117.

${ }^{11}$ N. W. Ashcroft and N. D. Mermin, Solid State Physics (Saunders, Philadelphia, 1976), pp. 1-27.

${ }^{12}$ Yu. G. Sadofyev, A. Ramamoorthy, B. Naser, J. P. Bird, S. R. Johnson, and Y.-H. Zhang, Appl. Phys. Lett. 81, 1833 (2002).

${ }^{13}$ T. Ando, in High Magnetic Fields in Semiconductor Physics II, edited by G. Landwehr (Springer, Berlin, 1989), pp. 164-173.

${ }^{14}$ W. Zawadzki, Adv. Phys. 23, 435 (1974).

${ }^{15}$ T. Ando, A. B. Fowler, and F. Stern, Rev. Mod. Phys. 54, 437 (1982).

${ }^{16}$ P. T. Coleridge, Phys. Rev. B 44, 3793 (1991). 